\title{
The Analysis of Application, Key Issues and the Future Development Trend of Blockchain Technology in the Insurance Industry
}

\author{
Lanqing Zhao \\ Bishop Allen Academy, Toronto, Canada \\ Email: zhaolanqing25@126.com
}

How to cite this paper: Zhao, L. Q. (2020). The Analysis of Application, Key Issues and the Future Development Trend of Blockchain Technology in the Insurance Industry. American Journal of Industrial and Business Management, 10, 305-314. https://doi.org/10.4236/ajibm.2020.102019

Received: January 8, 2020

Accepted: February 10, 2020

Published: February 13, 2020

Copyright () 2020 by author(s) and Scientific Research Publishing Inc. This work is licensed under the Creative Commons Attribution International License (CC BY 4.0).

http://creativecommons.org/licenses/by/4.0/

(c) (i) Open Access

\begin{abstract}
Blockchain, as an emerging technology, is essentially a shared database in which data or information is stored with features of "unforgeable", "whole-process traceable", "transparent" and "collective maintenance". Based on these characteristics, blockchain technology has a broad prospect of application in the insurance industry because it has laid a solid foundation of "trust", and created a reliable cooperation mechanism. At present, blockchain technology has been widely used in the fields of transportation, logistics, trade and charity. It has also attracted the attention of banking, insurance and other financial industries. The application of blockchain in other industries has been discussed in many other publications already, but it has not been systematically discussed in the insurance industry. This paper will make people have a deeper understanding of "blockchain + insurance" by introducing the basic concepts and technological advantages of blockchain technology, reviewing the researches and applications of blockchain by the insurance companies, and pointing out several key problems of applying blockchain technology to the insurance industry. Finally, the article will make predictions about the trend of development of applying blockchain technology in the insurance industry.
\end{abstract}

\section{Keywords}

Blockchain, Insurance, Smart Contract, Distributed Ledgers

\section{Introduction}

The buzz around blockchain technology stems from the rise of Bitcoin in 2008; it was seen as the most significant technological innovation of the generation after the internet. But most people have no idea how much of impacts it will have on the insurance industry. According to a recent study by PWC, blockchain could 
help to save $\$ 5$ billion - $\$ 10$ billion of cost for the insurance industry, in which accounting for 15 - 20 percent of the entire cost; however, nearly $57 \%$ of the respondents were still unaware of the potential impact and applicable opportunities of blockchain on them (Anonymous, 2016). On October 24, 2019, What the President of China, Xi, stressed further illustrates the importance of blockchain. He said that we should accelerate the development of blockchain technology and promote the integration of society and blockchain (Xinhuanet, 2019). Blockchain is a disruptive core technology, and it has completely changed the traditional way of value transmission. The application of blockchain in other industries has been discussed in many other publications already, but it has not been systematically discussed in the insurance industry. The method of argument used in this paper is the literature review method. This paper has shown that the application of blockchain technology has considerable advantages in reducing the operating cost of insurance companies, improving the efficiency of claims, protecting personal privacy, and avoiding "moral hazard". Reviewing the researches and applications about blockchain technology by insurance companies, we found that the main application field is automated claims, fraud identification and fund flow record tracking. However, the application of blockchain technology in the insurance industry is not yet mature because many fundamental problems have not been solved. At last, this paper stated the trend of development of blockchain technology in the insurance industry.

\section{The Significance and Development History of Blockchain}

Blockchain is a core technology including encryption algorithm, consensus algorithm, and sharing protocol. It enables all participant nodes to generate data synchronously and maintain data security jointly by making use of the characteristics of decentralization, distributed storage and smart contract. It has reconstructed the foundation of social credit in the Internet era, thus further constructed a new credit system, transaction system and rule system. China's research on blockchain technology is gradually maturing. China's ministry of industry and information technology released "China blockchain technology and application development white paper" in October 2016. This paper stated the critical path of blockchain technology development (The Chinese Academy of Industry Economy Research, 2016). In May 2017, the Ministry of industry and information technology released "Blockchain reference framework", it has established the necessary standards and clarified the direction of blockchain (Anonymous, 2017). So far, many provinces include Guangdong, Zhejiang, Jiangsu, Guizhou, Sichuan have issued guidelines on the development of blockchain technology; some provinces even listed blockchain technology in their 13th five-year plans. Besides, on the bases of the forecast by Gartner Group, the market value of blockchain will reach $\$ 176$ billion in 2025 and $\$ 3.1$ trillion in 2030 (Miller, 2019). All these examples illustrate the significance of blockchain for the future. Blockchain technology will revolutionize all aspects of our life. In the traditional social form, 
people used to let the social organizations established by the state to record and transmit every link of economic activities and chose to accept the registration of social organization unconditionally. Blockchain technology will rebuild the social credit foundation of today's society. In the future economic society, the status of social organization will gradually weaken from the middle core position.

Reviewing the development process of the blockchain, it mainly went through three stages, specifically as followings:

Blockchain 1.0: On November 1, 2008, Satoshi Nakamoto published "Bitcoin: a peer-to-peer electronic cash system", making the debut of blockchain technology (Satoshi, 2009). The first block with serial number "0" was born in Jan, 3nd 2009. A few days later, the block with serial number " 1 " appeared and was connected with the block with serial number "0", it officially marked the birth of blockchain.

Blockchain 2.0: Blockchain 2.0 has become the blockchain in a real sense that is programmable, which has added a series of protocols such as smart contracts based on the previous one. The so-called blockchain 2.0 era is ethereum, which supports Turing-complete languages. Ethereum provides the necessary infrastructure for developers to build the application on the "operating system" they set up, thus connect the virtual world with reality.

Blockchain 3.0: People can upload their assets onto the blockchain by blockchain 3.0 technology, because they can build a wide variety of applications within the underlying framework of a blockchain, thus creating a platform with no trust cost, low cost of risk and strong trading ability. This could enable the increasingly automated distribution of physical and human assets around the world, and facilitating large-scale collaboration in areas such as science, health and education. The blockchain 3.0 era could be a time full of significant changes because this technology can be extended to all aspects of daily life. With the establishment of many blockchain cities, any problems we confront can and will be readily solved.

\section{The Advantages of Blockchain Technology in Application}

First of all, blockchain technology can significantly reduce the operating costs of insurance companies because it can achieve permanent audit tracking. Blockchain is a decentralized distributed accounting system in which nodes can maintain a ledger together through a unified consensus mechanism without mutual trust, and each node has a complete data record. Therefore, in the environment of blockchain technology, transaction records cannot be tampered with, that is because the data based on the real transaction will be generated and uploaded by all participants simultaneously. At the same time, the blockchain's technical environment supports permanent data backtracking, any changes will be uploaded to the blockchain immediately after it was made, thus leaving no place to hide potential fraud; and eventually reduce the cost of building and maintaining trust that between the insurance company and customers. 
Second, blockchain technology can improve the efficiency of the insurance companies' claim. The initial claims process can be completely upgraded with the support of a smart contract. The smart contract exists in the blockchain technology itself. When the trading order in the blockchain touches the generating condition, the smart contract will automatically execute the set command independently. In contrast to the way the beneficiaries notify the insurance company after an accident, smart contracts can monitor a specific source of information through an oracle, and automatically notifying the insurers when they get certain information. Take life insurance, for example. The U.S. government has a file called the "Social Security Death Master File", which updates the death records of everyone who has U.S. security. Insurance companies can monitor it with a oracle, and when an insured person appears on the file, they can automatically start the claim process.

Third, blockchain can make personal privacy better protected. Although blockchain technology has the characteristics of information sharing, it will not affect privacy protection. Even if it is like a distributed database, all data retrieval and use must be done on the blockchain. This means that anyone who wants to retrieve a single user's data cannot bypass the user himself, on account of the retrieval process must be used with the user's authorization. In the blockchain environment, third parties will no longer rely on the certificates provided by participants, but directly enter the blockchain for viewing and supervision after the consent of participants'.

Fourth, blockchain technology will effectively decrease the existing "moral hazard" in the insurance industry. Currently, many customers take advantage of the asymmetry between the information owned by the insurance companies and their information to commit insurance fraud. Such customers file multiple claims against different insurers for unit losses. Some insurance companies, also make fraud together with the customers by taking advantages of the settlement that is not timely. Let us take the United States as an example, the FBI (Federal Bureau of Investigation) reports that insurance fraud totals more than $\$ 40$ billion annually, which means that insurance fraud costs the American family between $\$ 400$ to $\$ 700$ more a year in the form of increased premiums (Federal Bureau of Investigation, 2016).

\section{How Will Blockchain Technology Affect the Insurance Industry}

It has been centuries since the first insurance company was born in the $16^{\text {th }}$ century. However, the insurance companies still confront a lot of problems, such as misleading sales during the process of selling and claims disputes, etc., which result in a very strong sense of distrust in the insurance industry from all walks of the society, thus might restricting the development of insurance industry to some extent. The emergence of blockchain technology can reshape not only the foundation of the insurance industry but also reshape its image in the heart of 
the public. At the same time, it can help insurance institutions reduce operating costs. Specific, blockchain will have impacts on the insurance industry in the following areas.

1) Live transmission of information makes "one-stop settlement" possible. Blockchain requires that transaction information be generated and uploaded simultaneously. In the context of the continuous improvement of information transmission efficiency, the "one-stop settlement" long pursued by insurance will become a reality. All kinds of businesses handled by the industry that needs the participation of "insured + hospital + medical insurance department + insurance institution" will truly realize "one-stop settlement", such as serious disease insurance for urban and rural residents, and supplementary medical insurance for urban workers.

2) The unforgeable of transaction information can be used to protect the rights of all parties to the contract. Information about the insurance contract is scattered across all nodes, thus the policy-holder, the insured the insurer, and other participants have the same information. Neither party has the right to change the information that has been generated. Therefore, the rights and interests of both parties can be guaranteed. This plays a better role not only in mediating the contract disputes and protecting the legitimate rights and interests of consumers, but also fighting against illegal operations, false underwriting, false claims and other industrial chaos.

3) Uploading the underlying asset onto the blockchain will protect the legitimate rights and interests of all parties. With the popularization and application of $5 \mathrm{G}$ technology, online internet transaction will minimize the transaction costs and improve transmission efficiency. After the policy-holder signs a contract with the insurance company, the underlying asset will be uploaded to the blockchain immediately. At this point, all the parties to the contract can know the subject matter of the insurance; thus, many illegal behaviour such as repeated insurance, insurance fraud can be prevented.

\section{Application of Blockchain in the Insurance Industry at Present}

At present, the main application of blockchain in the insurance industry is "self-purchase insurance", "automatic claim settlement", "fraud identification", and "fund flow record tracking". More specific are as follows.

1) Previously, claims were mainly proceeded through KYC (know your customer), while automated claims de-intermediated KYC. KYC is one of the primary identification processes widely used by business organizations around the world and has been used by financial institutions since 2000. Customers can grant insurance companies to access to their identity data when needed. Once the KYC profile is verified, the customer can avoid duplicate authentication procedures and retrieve the verified identity data when needed by other companies. The KYC that based on blockchain technology has many advantages such as disintermediation, transparent transaction and no centralized control. 
Many enterprises have made use of the characteristics of de-mediation of blockchain to improve the way of information processing. For example, Stratumn, a insurance company based on Paris, France, shares the verified customer information through the blockchain, which saves the time and cost for each information demand-side to verify whether the customer meets the requirements for insurance purchase; thus the customers can purchase insurance independently (Jamie, 2016). Let us take another example. In December 2018, Ant Insurance launched the "blockchain + claim" project, in which the electronic notes can be used as claim notes from now on (Anonymous, 2018a).

Fraud detection mainly uses smart contract technology that is based on blockchain technology. Smart contracts are unique protocols designed to validate and enforce the contracts automatically. Specifically, smart contracts allow us to execute traceable, irreversible, and secure transactions without the need for third parties. A smart contract contains all the information about the transaction and will only perform the resulting operation if the requirements are met. The difference between smart contracts and traditional paper contracts is that smart contracts are generated by computers. Therefore, the code itself explains the relevant obligations of the participants. In essence, a smart contract is a digital contract that will produce no results unless the requirements are met. In fact, the participants in smart contracts are usually strangers on the Internet, so the digital agreements are more binding than the paper ones.

“Taikang Online" has had a project called "Anti-Moth" which uses blockchain technology to prevent insurance fraud. The intelligent contract system created by the company can identify whether the customer has the insurance intention and met the insurance qualification, which can not only protect the privacy but also prevent the insurance fraud at the same time (Anonymous, 2018b).

2) The nature of distributed ledgers makes it possible to track the records of capital flows over time. A distributed ledger is described as one that is maintained across multiple locations in a decentralized form and does not require a third party (such as a bank or clearinghouse) to maintain the authenticity of the data it holds. A distributed ledger is a database made from many independent computers (nodes); the nodes are responsible for verifying, saving, and updating information. The distributed ledger has the characteristics of distributed witness, which makes network attack extremely difficult. In a centralized ledger, only one entity holds a copy of the ledger. However, in a distributed ledger, all nodes of the network have a copy of the same ledger. No single entity may make changes to the ledger without the consent of all the participating nodes because any new changes will be added to all nodes within seconds. It uses cryptography to store all information and can only be unlocked using the key and encrypted signature. Therefore, the distributed ledger can not only realize the long-term tracking of capital flow records but also ensure the absolute security of the information stored.

Xinmei Life's "mutual insurance" project is the first "blockchain + mutual insurance" project in China. Through the blockchain technology, its account capi- 
tal flow is transparent. So that the data can be unchangeable and permanent for audit tracking (Zhou, 2019).

\section{Problems and Challenges for Applying Blockchain Technology to the Insurance Industry}

Currently, building insurance products on the blockchain is just a concept. The application still faces many governance and technical obstacles, mainly in the following four aspects.

1) There is still a long way to go to fully realize information sharing. The insurance market is an asymmetric market of the information in which each of the applicant and the insurer have their information that does not want to be known by each other. For example, when the customer is purchasing life insurance, the insurance companies always want to collect enough information about the insured's health, while the insured often wants to hide certain health conditions. Therefore, in order to achieve complete information sharing in medical insurance and other kinds of insurances, the whole society has to form a broad consensus on the blockchain construction. Therefore, achieving complete information sharing through blockchain technology still has a long way to go.

2) Different insurance products have different business models, such as life insurance and property insurance are operated differently. Many of these factors, such as business channels, actuarial models and customer group, will influence the operating earnings of the insurance institutions. Whether a specific insurance product is suitable for applying blockchain technology needs to be demonstrated independently, but the research on this aspect by the insurance companies is just beginning. Therefore, to promote the application of blockchain technology in the entire insurance industry needs time and orderly steps.

3) Due to the frequent occurrence of customer's fraud and the company's refusal in the insurance industry, there is a general distrust of insurance companies from the society. It is a fact that there is always a lack of trust between policy-holders, insurers and intermediaries in operation. While blockchain technology can simplify the process of buying insurance and making claims, it can exacerbate distrust. Therefore, insurance companies either need to do more work to build trust with customers or perfect the insurance mechanism in order to decrease credit cost.

4) Most insurance companies have a limited understanding of blockchain and have not yet made clear how blockchain can strategically help their business development. They generally lack the capacity to carry out pilot projects and improve production practices.

5) Although blockchain can connect the insurance companies with other related industries, however, we have to emphasize that the insurance industry is connected to everything from health care to education to even business operation. Therefore, to build a complete blockchain requires high connection cost and tremendous amount of works. 


\section{Prospects of the Combination of Blockchain and Insurance}

Blockchain technology is gradually changing people's traditional mode of thinking; it has spawned many new business models. At present, some insurance companies have already realized the importance of blockchain technology. In the future, the model of "blockchain + insurance" will be applied in a higher level and broader field in the insurance industry. Followings are the predictions of the development mode of "blockchain + insurance".

1) The authentication of the customer's identity through shared information. At present, employees or agents in the insurance industry often take the place of the customers to receive the surrender money or survival money, which is illegal and violated the interest of the customers'. What caused that problem is that the insurance institutions do not accurately identify the identity of the customer. If a blockchain identity is granted to the customer, the customer's identity is no longer determined by the citizen ID card but needs to be verified by all parties involved, which will largely eliminate the risk of all kinds of judicial cases in the industry.

2) Establishing industry blacklist by depositing certificated data. At present, the employment requirements of the insurance industry are relatively low, which leads to a large number of agents who violated the principle of good faith in the industry. At the same time, there are also many clients who are the subject of criminal cases. However, due to the lack of blacklist platform in the industry, it is impossible to identify the employees and customers effectively. The establishment of industry blacklist based on the blockchain data storage technology will play a better role in cracking down on insurance fraud and other illegal behaviour.

3) Preventing false claims by uploading the underlying property information to the blockchain. In the property insurance contract, the underwriter often loses control of the actual situation of the underlying property after the contract is the signer. Many types of insurance are prone to overpayment due to lack of control, such as cargo insurance, art insurance and warranty insurance. By uploading the underlying assets to the blockchain, the underlying asset can be tracked in the whole process, to protect the interests of both parties. This will effectively prevent the risk of repeated insurance, property control and false claims.

4) Making claims automatically through smart contracts. In the claim settlement procedure, all information and data will no longer be required from the occurrence of the accident to the payment of the indemnity. It will no longer be necessary to check, determine the loss, make valuation and other procedures, but will be completed automatically through the intelligent contract. For example, when a vehicle has had an accident, the information of the accident can be automatically collected and uploaded to the insurance company. Insurance companies will pay the claims automatically after receiving the instructions; thus, faster claims will be achieved. It will not only save labour costs but also improve the customer's satisfaction. 
5) The application of the tracking technology that is based on blockchain technology will improve the mechanism of mutual insurance. The main reason that restricts the development of mutual insurance lies in the distrust of the insurance companies by the customers because customers cannot know the direction of each fund of their mutual insurance companies. However, the information traceability technology of blockchain can ensure that participants know the whereabouts of each fund so that the participants can fully trust the mutual insurance company. Therefore, mutual insurance companies will grow rapidly in an environment of trust.

In general, although blockchain technology is still in its infancy, it has many promising application methods in the insurance industry. In the future, blockchain technology will integrate with the insurance under the guidance of the government and will play a more significant role in serving the real economy and preventing financial risks.

\section{Conclusion}

As an emerging technology, blockchain can achieve distributed accounting, asymmetric encryption and smart contracts. It has played a significant role in reducing the operating cost of insurance companies, improving the efficiency of claims, protecting personal privacy and avoiding "moral hazard". At present, the research and application of blockchain technology by the insurance institutions are still very few, mainly for automated claims, fraud identification and fund flow tracking. The application of blockchain technology in the insurance industry is not yet mature, and many important problems have not been solved. This paper summarizes the current application of blockchain technology in the insurance industry, and on this basis predicts several ways of blockchain technology overturn the operation mode of the traditional insurance industry. However, with the rapid development of science and technology, many insurance companies have realized the significance of blockchain technology. We believe in the future, "Blockchain + Insurance" will be applied in a higher level and broader field in the insurance industry.

\section{Conflicts of Interest}

The author declares no conflicts of interest regarding the publication of this paper.

\section{References}

Anonymous (2016). PWC: Blockchain Will Revolutionize the Insurance Industry. http://www.sohu.com/a/110229804_286863

Anonymous (2017). Miit Releases China's First Blockchain Standard, Blockchain Reference Architecture. https://www.doit.com.cn/p/274630.html

Anonymous (2018a). Ant Financial Has Completed the Country's First Blockchain Insurance Claim.

https://baijiahao.baidu.com/s?id=1619543570005996432\&wfr=spider\&for=pc 
Anonymous (2018b). Taikang Online Launched "Anti-Moth" Project as the First Attempt at the Insurance Industry Blockchain.

https://baijiahao.baidu.com/s?id=1597330997391735750\&wfr $=$ spider\&for $=\mathrm{pc}$

Federal Bureau of Investigation (2016). Insurance Fraud. https://www.fbi.gov/stats-services/publications/insurance-fraud

Jamie, R. (2016). Meet Lender Bot: Stratumn \& Deloitte's Blockchain Insurance Bot. https://news.bitcoin.com/stratumn-deloitte-blockchain-bot/

Miller, M. (2019). Gartner's Top 10 Strategic Technology Trends for 2019. https://au.pcmag.com/forward-thinking/59160/gartners-top-10-strategic-technology-tr ends

Satoshi, N. (2009). Bitcoin: A Peer-to-Peer Electronic Cash System. https://bitcoin.org/en/bitcoin-paper

The Chinese Academy of Industry Economy Research (2016). The Ministry of Industry and Information Technology Released White Paper on China's Blockchain Technology and Application Development 2016.

http://www.askci.com/news/finance/20161020/08575771031.shtml

Xinhuanet (2019). During the 18th Joint Study Session of the Political Bureau of the Communist Party of China (CPC) Central Committee, Xi Jinping Stressed the Importance of Blockchain as a Significant Breakthrough in Independent Innovation of Core Technologies to Accelerate the Development of Blockchain Technology and Industrial Innovation. http://www.xinhuanet.com/politics/2019-10/25/c_1125153665.htm

Zhou, L. (2019). Blockchain Technology Has Been Officially Launched in the Field of Xinmei Life Insurance Co., LTD. http://www.elecfans.com/blockchain/934708.html 